\title{
Formación en oncología urológica laparoscópica. La experiencia del Memorial Sloan-Kettering Cancer Center
}

\author{
Touijer K, Guillonneau B. \\ Sidney Kimmel Center for Prostate \& Urologic Cancers. Memorial Sloan-Kettering Cancer Center, New York, \\ Actas Urol Esp 2006; 30 (5): 464-468
}

\section{RESUMEN \\ FORMACIÓN EN ONCOLOGÍA UROLÓGICA LAPAROSCÓPICA. LA EXPERIENCIA DEL MEMORIAL SLOAN-KETTERING CANCER CENTER}

Objetivo: Presentar un breve esquema de la estructura del programa clínico y de formación en oncología urológica laparoscópica en el Memorial Sloan-Kettering Cancer Center. Describir las fases y los elementos claves necesarios para adquirir la capacitación en laparoscopia.

Material y métodos: El programa dura 2 años y forma a residentes en formación y a urólogos. En el caso de los residentes, el programa consiste en una rotación en oncología con un gran volumen de procedimientos laparoscópicos, de 6 meses de duración, en el cual se adquiere experiencia en laboratorio de simulación, laboratorio animal, revisión de vídeos y experiencia en quirófano. Para los urólogos, el programa consiste en 1 curso de formación médica continuada acreditada, 20 horas de laboratorio de simulación, 1 sesión de laboratorio animal, observación de casos laparoscópicos, ejercer como primer ayudante en un mínimo de 15 procedimientos laparoscópicos y ejecución de procedimientos laparoscópicos bajo supervisión.

Resultados: 8 residentes han completado el programa de formación laparoscópica, 4 de los cuales han completado su ciclo de especialización y están en centros académicos, realizando laparoscopia avanzada. El abordaje supone un promedio del $80 \%$ de su práctica urológica. Tres de los participantes están realizando cirugía laparoscópica bajo supervisión.

Conclusión: Los objetivos de un programa de formación quirúrgica deberían ser la normalización de la adquisición de experiencia quirúrgica y la evaluación de los resultados en un marco uniforme para garantizar la conservación de la experiencia adquirida y desarrollar programas para enseñar nuevas técnicas.

Palabras clave: Formación. Laparoscopia. Urología oncológica.

\section{ABSTRACT \\ TEACHING LAPAROSCOPIC UROLOGIC ONCOLOGY. THE MEMORIAL SLOAN-KETTERING CANCER CENTER EXPERIENCE}

Objective: We outline the structure of the clinical and training program of laparoscopic urologic oncology at Memorial Sloan-Kettering Cancer Center. We discuss the steps and key elements necessary in acquiring laparoscopic proficiency.

Material and Method: The program lasts 2 years and trains fellows and faculty. For fellows, the program consists of a 6 months high volume laparoscopic oncology rotation, during which dry lab, animal lab, video review and operating room experience are required. For faculty, the program consists of 1 accredited continuing medical education course, 20 hours of dry lab, 1 session animal lab, observation of laparoscopic cases, first assistant in a minimum of 15 laparoscopic cases, performing laparoscopic cases under mentoring.

Results: 8 fellows have completed the training, 4 of whom have completed their fellowship and are in academic centers, performing advanced laparoscopy. The laparoscopic approach represents on average 80\% of their urologic practice. Three attendings are performing laparoscopic surgery with mentoring.

Conclusion: The goals of a surgical education program should be the standardization of the acquisition of surgical skills and assessment of the performance in a uniform setting to ensure the maintenance of the acquisition of skills and to develop programs to teach new skills.

Keywords: Teaching. Laparoscopy. Urologic oncology. 
Como especialidad quirúrgica, la urologia se ha adaptado totalmente a la llegada de la laparoscopia. En el pasado, los urólogos ya demostraron estar abiertos a los nuevos métodos mínimamente invasivos, como la endoscopia, que revolucionó los paradigmas del tratamiento de la litiasis y de la uropatía obstructiva. Actualmente, la laparoscopia se está haciendo cada vez más popular entre pacientes y cirujanos por igual y pronto se convertirá, si es que no lo es ya, en el método estándar de intervención en muchas enfermedades urológicas. Sin embargo, este rápido éxito en su aplicación debe ir de la mano de un éxito en la reproducibilidad y en la capacidad de ser enseñada. La adquisición de una experiencia adecuada de un modo eficiente en cuanto al tiempo está siendo cada vez más difícil. Las limitaciones económicas y médico-legales han limitado las oportunidades del médico en prácticas de aprender y adquirir experiencia mientras opera a pacientes reales. Nuestro reto y responsabilidad como profesores de cirugía frente a nuestros pacientes y médicos en prácticas es ofrecer atención y enseñar sin comprometer la calidad de ninguno de los dos aspectos. En este documento, describimos en líneas generales la estructura del programa clínico y de formación en oncología urológica laparoscópica en el Memorial SloanKettering Cancer Center y discutimos las diferentes fases y elementos claves necesarios para adquirir experiencia en laparoscopia.

\section{LA EXPERIENCIA DEL MEMORIAL SLOAN-KETTERING CANCER CENTER}

El programa establecido en el Memorial SloanKettering Cancer Center durante los 2 últimos años va dirigido a la formación de residentes y urólogos para la adquisición de la experiencia necesaria para realizar procedimientos de laparoscopia.

\section{Residentes}

El programa consiste en una rotación en oncología con un gran volumen de procedimientos laparoscópicos, de 6 meses de duración, en el cual se adquiere experiencia en laboratorio de simulación, laboratorio animal, revisión de vídeos y experiencia en quirófano. Los médicos intervienen en la asistencia preoperatoria, intraoperato- ria y posoperatoria de los pacientes. En cada período docente se forma a 2 residentess que asistirán a diversos tipos de cirugía laparoscópica oncológica, incluyendo entre otras prostatectomía radical, nefrectomía y adrenalectomía radical o parcial. Por término medio cada semana se realizan de 6 a 8 intervenciones laparoscópicas mayores.

Se promueve la participación activa de los residentes en el laboratorio de simulación, en el laboratorio animal y la revisión de la grabación de las operaciones antes y durante su rotación clínica. En el laboratorio de simulación, se utiliza un "pelvi-trainer" para dominar la percepción en profundidad, superar el efecto fulcro y aprender técnicas de sutura y manipulación de tejidos realizando una simulación de una anastomosis uretrovesical. La revisión de las grabaciones de vídeo y el elevado volumen de prácticas permite a los participantes aprender las diferentes fases de una operación, permite la repetición frecuente de ejercicios similares y muestra distintas situaciones con diferentes grados de dificultad, pero lo que es más importante representa una oportunidad para aprender los principios y los matices de la cirugía oncológica.

\section{Urólogos}

Las siguientes directrices son los requisitos mínimos para los urólogos que deseen continuar con la especialización en procedimientos laparoscópicos, y pueden variar individualmente de acuerdo con los intereses particulares de cada uno.

La especialización abarca sólo un tipo de cirugía, o bien cirugía de las vías urinarias superiores (adrenalectomía, nefrectomía radical y nefrourectomía) o cirugía pélvica (prostatectomía radical).

1. Un curso de formación médica continuada acreditada [animal o cadáver].

2. Veinte horas de trabajo en el laboratorio de simulación.

a. Simulación.

b. Modelo Pelvi-trainer.

c. Modelo de perfusión de órganos.

3. Una sesión de trabajo en el laboratorio animal.

a. Se requieren procedimientos ablativos y reconstructivos. 
4. Observación de casos laparoscópicos (quirófano y vídeo).
a. Riñón-mínimo 10 casos (5 derechos/5 izquierdos, radical y parcial).

b. Próstata-mínimo 15 casos.

c. Es muy recomendable la observación de casos en otros centros.

5. Primer ayudante en un mínimo de 15 casos laparoscópico.

6. Realización de procedimientos laparoscópicos bajo supervisión.

a. El número de procedimientos laparoscópicos se adaptará a cada cirujano.

1. Las fases 1 a 4 se pueden completar simultáneamente.

2. Las fases 5 y 6 se completarán consecutivamente.

\section{Resultados}

Hasta la fecha, 8 residentes han completado el programa de formación laparoscópica, 4 de los cuales han completado su ciclo de especialización y están en centros académicos, realizando laparoscopia avanzada. El método laparoscópico representa un promedio del $80 \%$ de su práctica urológica (cifra que varía entre el 30\% y el 90\%) dependiendo de las demandas de sus respectivas instituciones.

Tres de los participantes están realizando cirugía laparoscópica bajo supervisión, 2 de ellos cirugía pélvica y uno laparoscopia de las vías urinarias superiores.

\section{DISCUSIóN}

Los retos del aprendizaje de la laparoscopia

Al contrario de lo que sucede con los procedimientos de cirugía abierta, la laparoscopia conlleva varios cambios en lo que se refiere a la forma en que se realiza la cirugía. La coordinación manual-visual está alterada, la sensación táctil está disminuida, los grados manuales de libertad están limitados y se pierde la vista tridimensional. Estos retos constituyen los obstáculos iniciales de la laparoscopia y su superación constituye el primer paso en el aprendizaje de la misma. Esto se consigue generalmente mediante la práctica repetitiva en el laboratorio y por la cual el cirujano adquirirá las habilidades básicas y la destreza quirúrgica.
El siguiente paso del aprendizaje es la exposición clínica a una amplia variedad de procedimientos quirúrgicos y la adquisición de conocimientos sobre los diferentes pasos y su complejidad. Durante esta fase, el participante adquiere las habilidades psicomotoras, comenzando por aprender los pasos básicos de la "fase cognitiva" del procedimiento. En segundo lugar, tiene lugar la transferencia a las habilidades psicomotoras y, por último, la fase automática, donde mediante la práctica repetitiva se perfeccionan las habilidades motoras, de modo que el procedimiento se ejecuta automáticamente sin apenas intervención cognitiva. Esta fase se enseña en el laboratorio de simulación, en el laboratorio animal, en la videoteca y en el quirófano.

La tercera fase es crucial puesto que es la esencia de la ejecución de los procedimientos laparoscópicos. Es la interpretación filosófica de los procedimientos, el grado de dificultad de cada paso, los pasos que requieren un máxima atención, entender que el método laparoscópico es implacable y que la maestría se consigue evitando complicaciones críticas más que intentando superar un resultado complicado. El papel del profesor durante esta fase es de la máxima importancia.

\section{Establecimiento de un programa de formación estructurado}

En el actual panorama urológico, es necesario establecer un programa de formación laparoscópica por muchas razones: la laparoscopia es ligeramente más difícil de ejecutar que la cirugía abierta, los urólogos formados a principios de los 90 tuvieron una exposición mínima o nula a la laparoscopia y existe una falta de rutina de procedimientos laparoscópicos urológicos (como el caso de la colecistomía en los cirujanos generales). El establecimiento de un programa de formación de éxito tiene que contemplar los siguiente puntos: 1- formación de urólogos, 2- formación de residentes, 3- ofrecer directrices para la formación en la fase posterior a la residencia.

En este proceso de formación gradual, y en primer lugar, se aprenden las habilidades psicomotoras en el laboratorio de simulación. Los elementos claves de un período de formación exitosa en el laboratorio de simulación son: repetición, práctica regular y, lo que es más importante, la 
propia motivación. Las habilidades psicomotoras básicas se pueden adquirir fácilmente con un sencillo pelvi-trainer, un simulador sencillo que puede conseguirse sin problema. Este simulador permite el uso de los mismos instrumentos y ejercicios quirúrgicos, como la sutura o la realización de la anastomosis uretrovesical, siendo un instrumento excelente porque incorpora la percepción en profundidad, la manipulación de tejidos a través de la resistencia a la tracción de los nudos, a la vez que el alumno se centra en la calidad del resultado final. Sin embargo, al contrario de lo que sucede con los simuladores mecánicos, informatizados o híbridos, el pelvi-trainer no ofrece ninguna evaluación y no recrea la anatomía humana, por lo que es prácticamente imposible que dé impresión de realidad. No obstante, las habilidades adquiridas en el laboratorio de simulación se pueden transferir al laboratorio animal, donde el alumno aprenderá el control hemostático, la manipulación de tejidos y la disección vascular en un modelo animal que muchas veces es similar al cuerpo humano, con la salvedad de que el modelo animal no ofrece las mismas dificultades específicas que las encontradas en el ser humano, especialmente en procedimientos como la nefrectomía.

En segundo lugar está la exposición clínica, durante la cual el alumno pasa por diferentes fases de aprendizaje comenzando con la Fase cognitiva, donde aprende los pasos del procedimiento revisando grabaciones de vídeo y ayudando con frecuencia y regularidad a un cirujano experto antes de pasar a la fase de integración, la cual consiste en una combinación de las habilidades psicomotoras adquiridas en el laboratorio y las habilidades cognitivas adquiridas durante su intervención como ayudante en el quirófano. Y, por último, la fase automática se alcanza cuando ya no es necesaria la intervención cognitiva y los pasos se ejecutan automáticamente y se convierten en naturales. En un estudio cuyo objetivo era la identificación de los factores que contribuyen a diferenciar a los alumnos de los cirujanos experimentados, Gupta et al., identificaron como factores principales la falta de conocimiento de estas fases y la falta de sincronización del movimiento con la consiguiente fatiga temprana, llegando a la conclusión de que es importante incorporar un trabajo intensivo en el laboratorio de simulación dentro del programa de formación ${ }^{1}$.

La finalización con éxito de la fase automática es solamente la conclusión de la enseñanza técnica de la laparoscopia. Un programa de formación laparoscópica debería ir más allá de la fase automática y ofrecer una sólida supervisión para ayudar al alumno a descubrir el esfuerzo y los requerimientos especiales de cada procedimiento, reconocer sus propias limitaciones, las limitaciones de la propia tecnología, y por último, reconocer que la laparoscopia es solamente un procedimiento y que la enfermedad, las indicaciones y los principios en los que se basan los objetivos del tratamiento son los mismos.

¿Cómo definimos y evaluamos la capacitación?

La definición de capacitación quirúrgica es un debate actual complejo, ya que la capacitación es dificil de medir. Por otro lado, las habilidades quirúrgicas se pueden medir por la velocidad o rapidez, la ausencia de errores técnicos y la seguridad. Se han propuesto varias pruebas objetivas y se ha intentado medir las habilidades o tareas quirúrgicas mediante el Sistema inanimado McGill para la enseñanza y evaluación de las habilidades laparoscópicas, MISTELS (acrónimo de McGill Inanimate System for Training and Evaluation of Laparoscopic skills), el cual tiene en cuenta la velocidad y precisión en la ejecución de una serie de tareas laparoscópicas ${ }^{2}$. Otros sistemas como la Evaluación estructurada objetiva de las habilidades técnicas, OSATS (Objective Structured Assessment of Technique Skills), o el Ensayo psicomotor avanzado de Dundee, ADEPT (Advanced Dundee Psychomotor Test), miden la capacidad psicomotora básica y algunos han propuesto que se incorporen en la selección inicial de los alumnos que potencialmente fueran más aptos para una carrera quirúrgica basándose en el concepto de aptitud innata ${ }^{3,4}$.

Por otro lado, la capacitación quirúrgica podría asimilarse a la calidad quirúrgica $\mathrm{y}$, por consiguiente, podría medirse individualmente para cada tipo de procedimiento. En el caso de la prostatectomía radical, los indicadores de calidad podrían ser, el porcentaje de márgenes quirúrgicos positivos, el porcentaje de recuperación de potencia y continencia y el perfil de complicacio- 
nes, en el caso de la nefrectomía parcial laparoscópica también podrían utilizarse los márgenes quirúrgicos positivos, el tiempo de isquemia, el porcentaje de fístulas urinarias y la hemorragia perioperatoria.

\section{CONCLUSIONES}

La aplicación de la laparoscopia en urología se está haciendo cada vez más popular entre pacientes y cirujanos por igual, lo cual requiere su completa integración en todo programa de formación en urología. Los objetivos de un programa de formación quirúrgica deberían ser la normalización de la adquisición de experiencia quirúrgica y la evaluación de los resultados en un marco uniforme para garantizar la conservación de la experiencia adquirida y desarrollar programas para enseñar nuevas técnicas.

\section{FASES EN EL APRENDIZAJE DE LA} LAPAROSCOPIA

1. Curso de formación básico y avanzado.

2. Pelvi-Trainer.

3. Visita a centros con un elevado volumen y reputación internacional.
4. Colaboración con el cirujano experto.

5. Realización de procedimientos bajo supervisión de un cirujano experto.

6. Realización de procedimientos de forma independiente.

7. Siempre se trata de un procedimiento en equipo.

\section{REFERENCIAS}

1. Gupta R, Cathelineau X, Rozet F, Vallacien G. Feedback from operative performance to improve training program of laparoscopic radical prostatectomy. J Endourol. 2004; 18(9): 836-839.

2. Derossis AM, Antoniuk M, Fried GM. Evaluation of laparoscopic skills: a 2-year follow-up during residency training. Can J Surg. 1999 Aug;42(4):293-296.

3. Martin JA, Regehr G, Reznick R, MacRae H, Murnaghan J, Hutchison C, et al. Objective structured assessment of technical skill (OSATS) for surgical residents. Br J Surg. 1997; 84(2):273-278.

4. Schijven MP, Jakimowicz J, Schot C. The Advanced Dundee Endoscopic Psychomotor Tester (ADEPT) objectifying subjective psychomotor test performance. Surg Endosc. 2002; 16(6):943-948.

Dr. D. K. Touijer

Sidney Kimmel Center for Prostate \& Urologic

Cancers. Memorial Sloan-Kettering Cancer Center, New York 